\title{
Ostwald Ripening of Platinum Nanoparticles Confined in a Carbon Nanotube/Silica-Templated Cylindrical Space
}

\author{
Cintia Mateo-Mateo, ${ }^{1}$ Carmen Vázquez-Vázquez, ${ }^{1}$ Moisés Pérez-Lorenzo, ${ }^{1}$ \\ Verónica Salgueiriño, ${ }^{2}$ and Miguel A. Correa-Duarte ${ }^{1}$ \\ ${ }^{1}$ Departamento de Química Física, Universidade de Vigo, 36310 Vigo, Spain \\ ${ }^{2}$ Departamento de Física Aplicada, Universidade de Vigo, 36310 Vigo, Spain \\ Correspondence should be addressed to \\ Verónica Salgueiriño,vsalgue@uvigo.es and Miguel A. Correa-Duarte, macorrea@uvigo.es
}

Received 7 July 2012; Revised 2 October 2012; Accepted 2 October 2012

Academic Editor: Steve F. A. Acquah

Copyright (C) 2012 Cintia Mateo-Mateo et al. This is an open access article distributed under the Creative Commons Attribution License, which permits unrestricted use, distribution, and reproduction in any medium, provided the original work is properly cited.

\begin{abstract}
Sintering of nanoparticles mediated by an Ostwald ripening mechanism is generally assessed examining the final particle size distributions. Based on this methodology, a general approach for depositing platinum nanoparticles onto carbon nanotubes in solution has been employed in order to evaluate the sintering process of these metallic nanoparticles at increasing temperatures in a carbon nanotube/silica-templated confined space.
\end{abstract}

\section{Introduction}

Nanoparticles (NPs) are inherently unstable due to their high surface area and therefore tend to grow and increase their average size $[1,2]$. Sintering refers to the increase in mean particle size that occurs as a system of NPs evolves to attain a lower-energy state [3]. The driving force in a process of sintering of NPs corresponds to their increased surface free energy, if compared with bulk, by which NPs present a metastable solid state and inevitably tend to aggregate into larger nanostructures [2].

Sintering is an acute drawback in catalysis, especially if working at elevated temperatures. In the case of supported catalysts, sintering is typically attributed to mass-transport mechanisms involving atomic migration to subsequent coalescence with neighboring NPs. The kinetic models proposed have been established considering an Ostwald ripening, by which the migration mechanism refers to diffusion of atoms between immobile NPs either on the surface of the support or through the gas phase [4]. In these conditions, the concentration of atomic species is higher in the vicinity of small particles than of large particles. There is, consequently, a concentration gradient that leads to a net flux of atomic species from the smaller particles toward the larger ones, so the larger particles eventually grow at the expense of the smaller ones. Accordingly, Yang et al. studied the sintering of $\mathrm{Au}$ NPs on $\mathrm{TiO}_{2}$ and were able to explain the observed evolution of particle sizes using a ripening model [5], and Simonsen et al. confirmed that Ostwald ripening is responsible for the sintering of Pt under oxidative atmospheres [6]. Since Pt is known to form a volatile oxide, they claimed as plausible that this Pt oxide constitutes the mobile phase responsible for Pt sintering.

In the Ostwald ripening process, the atomic species are exchanged among immobile NPs driven by a difference in their chemical potential, as described by the GibbsThomson equation. In addition to NP size, parameters such as morphology of the NPs and NP-support interactions will also play a role in the sintering process [2]. Additionally, given the increasing importance of reactions in restricted dimensions such as Pt catalyst-bearing nanoreactors (e.g., nanocapsules) [7], the influence of confined spaces on the Ostwald ripening should be carefully assessed given its critical influence on the catalytic efficiency of metallic nanoparticles. 
Herein, we engineer the deposition of small Pt nanoparticles on one-dimensional CNT templates and their later encapsulation with an inorganic silica shell. Such a system was developed to study the sintering of Pt NPs in specific conditions given by elevated temperatures and the confined cylindrical nanospace given to the Pt NPs to grow. The direct observation of sintering in these extreme conditions is demonstrated in terms of an evolution of the average size distribution from Gaussian to log-normal.

\section{Experimental Section}

2.1. Polymer-Wrapping Functionalization of CNTs [8]. CNTs (MWNTs from Nanolab, 5-15 $\mu \mathrm{m}$ length and 10-15 nm diameter, $95 \%$ purity) were redispersed in ultrapure water $(18 \mathrm{M} \Omega-\mathrm{cm})$ according to the following procedure. Briefly, CNTs were dispersed in a $1 \mathrm{wt}$ \% aqueous solution of pollyallylamine hydrochloride ( $\mathrm{PAH}, 0.5 \mathrm{M} \mathrm{NaCl}, \mathrm{pH}=10)$ up to a concentration of $150 \mathrm{mg} / \mathrm{L}$. A combination of rapid stirring and sonication was used to ensure the presence of individually well-dispersed CNTs (this is demonstrated by the fact that CNTs appear individually coated with Pt NPs and subsequently with the outer silica shell (vide infra)). Excess of PAH was removed by three centrifugation cycles at $9000 \mathrm{rpm}$, at $25^{\circ} \mathrm{C}$ during $12 \mathrm{~h}$ to be later redispersed in aqueous solution.

2.2. Synthesis of Platinum NPs [7]. Pt NPs were synthesized as follows: to a solution containing $43 \mathrm{~mL}$ of ultrapure water, $2.5 \mathrm{~mL}$ of sodium citrate $(0.1 \mathrm{M})$ and $2.5 \mathrm{~mL}$ of $0.05 \mathrm{M}$ $\mathrm{H}_{2} \mathrm{PtCl}_{6}$, and $2.45 \mathrm{~mL}$ of sodium borohydride $(0.015 \mathrm{M})$ were added as reducing agent (citrate $/ \mathrm{H}_{2} \mathrm{PtCl}_{6} / \mathrm{NaBH}_{4}$ in a molar ratio of $2: 1: 0.3$ ). The solution was stirred for $10 \mathrm{~min}$.

2.3. NPs Deposition onto CNTs [7]. CNTs@PAH (12 mL, $0.016 \mathrm{mg} / \mathrm{mL})$ were added to $50 \mathrm{~mL}$ of Pt NPs $(0.5 \mathrm{mM})$. After $1 \mathrm{~h}$, the solution was centrifuged three times $(3500 \mathrm{rpm}$, RT, $20 \mathrm{~min}$ ) and redispersed in $20 \mathrm{~mL}$ of ultrapure water removing the nonadsorbed Pt NPs after centrifuging.

2.4. Silica Coating of CNTs@Pt. Silicon oxide was deposited on the CNTs@Pt nanocomposites using two different steps [9]. In the first silica-deposition step, the hydrolysis and condensation of 3-aminopropyltrimethoxysilane (APS) and tetraethoxysilane (TEOS) were carried out under acidic conditions. Ethanol $(20 \mathrm{~mL})$ containing APS and TEOS (typically $2.88 \mu \mathrm{L}$ APS and $33.8 \mu \mathrm{L}$ TEOS) was added dropwise to $10 \mathrm{~mL}$ of an aqueous dispersion of CNTs@Pt $(0.03 \mathrm{mg} / \mathrm{mL})$ containing citric acid $(10 \mathrm{mM}, \mathrm{pH} \sim 3)$ under magnetic stirring. In the second step and after stirring for $2 \mathrm{~h}$ $30 \mathrm{~min}$, the $\mathrm{pH}$ value of the solution was rapidly increased so that the silane condensation was carried out under basic conditions. Therefore, a solution of $\mathrm{NH}_{4} \mathrm{OH}(0.01 \mathrm{M})$ was added dropwise until a $\mathrm{pH}$ value of 8-10 was reached. Subsequently, the solution was aged for $3 \mathrm{~h}$ and a compact, uniform, $15 \mathrm{~nm}$ thick silica shell was obtained. Subsequently, the solution was centrifuged $(950 \mathrm{rpm}, 10 \mathrm{~min}, \mathrm{RT})$ and redispersed three times in $\mathrm{EtOH}$.
2.5. Sintering of CNTs@Pt@SiO 2 . Thermogravimetric Analysis (TGA) of CNTs coated with Pt NPs and silica shell was carried out heating up to $830^{\circ} \mathrm{C}$. The sintering process was studied heating up the composite nanostructures at two temperatures $\left(550\right.$ and $\left.800^{\circ} \mathrm{C}\right)$ in order to tune the NP agglomeration.

\section{Results and Discussion}

CNTs are some of the most intensively explored nanostructured materials. Their unique properties render these structures as ideal templates for the design of nanosized architectures [10]. In this case, their uniform curvature allows the deposition of Pt NPs to be further coated with silica, in an effort to isolate them in a cylindrical and therefore curved confined space. Accordingly, a general approach for depositing NPs onto CNTs in solution has been employed [8]. This approach, with an initial step based on the polymer wrapping technique, allows the manipulation of CNTs in solution. The polymer wrapping method, developed by O'Connell et al. [11], stems from a functionalization of CNTs through noncovalent attachment. This technique is based on the thermodynamic preference of CNT-polymer interactions over CNT-water interactions, which favors the hiding of the hydrophobic surface of CNTs in aqueous solution, giving rise to a better dispersion $[12,13]$. In this way, CNTs can be wrapped either with a negatively or positively charged polyelectrolyte (PE) depending on the surface charge of the metallic nanoparticles to be deposited. The polymer layer allows a homogenous high-density coverage of charges on the tips and walls of CNTs and, as result, an efficient deposition of the metallic nanoparticles through electrostatic interactions. A further synthetic approach consists of the use of this initial polymer layer as a primer for adsorption of successive oppositely charged PE layers. Thus, an ordered polymer multilayer around the carbon nanotube is obtained. Through this method, better known as layer-by-layer assembly technique (LbL) [14], an easy and exact modulation of the polymer shell thickness can be attained by controlling the number of deposition steps. This technique has indeed been demonstrated to be very efficient not only for the coating of CNTs with different types of NPs [12] but also for tuning the distance in between [15].

In our case, Pt NPs were driven to the surface of the CNTs taking advantage of the PE (PAH) previously deposited on the surface of these supports. Because of the positively charged surface provided by this polymer, these NPs were easily attached becoming fixed in the final composite nanostructure. It must be noted that the Pt NPs employed were previously synthesized in aqueous solution, reducing $\mathrm{H}_{2} \mathrm{PtCl}_{6}$ salt with sodium borohydride and stabilizing them with citrate ions, which confers them a negatively charged surface. Figure 1 shows TEM images at different magnification of the CNTs coated with the Pt NPs, very homogeneously distributed all over the surface of the carbon nanostructures. It is also worthy to mention the pretty narrow size distribution of such small metallic nanoparticles, centered at $2.62 \pm 0.60 \mathrm{~nm}$ (95\% of NPs). Without a doubt, 


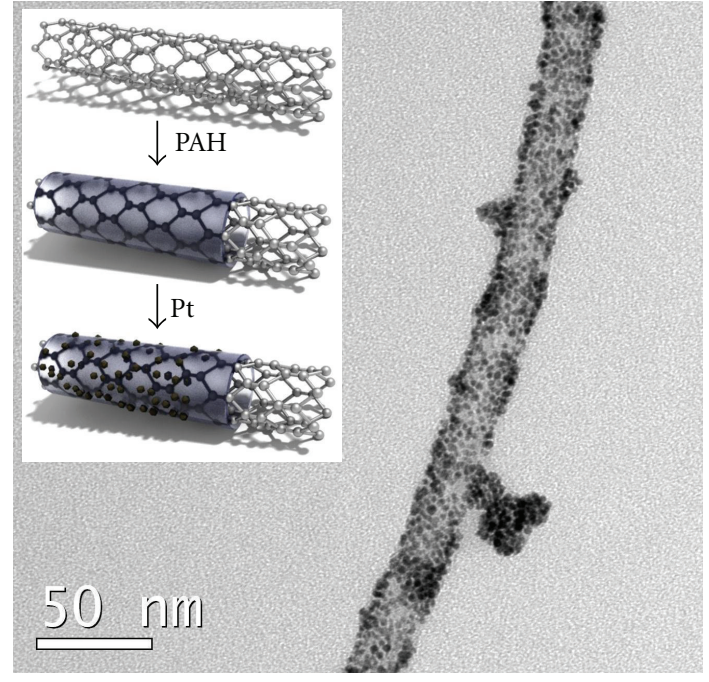

Figure 1: TEM image of a representative CNT coated with $\mathrm{Pt}$ nanoparticles homogeneously distributed on their surface (inset: scheme of the process employed to drive first the PAH and later the Pt NPs to the surface of CNTs).

it is also important to draw attention to the aggregates of Pt nanoparticles, assembled during the process and located or distributed all along every CNT. These aggregates of NPs were likely formed once the positively charged CNT solution was added, considering the fact that it may carry different ions necessary for the assembly of the PE layer and may consequently increase the ionic strength. These conditions can therefore explain some previous aggregation of the NPs, which, however, does not prevent their homogeneous deposition onto the CNTs.

As next step, these composite nanostructures were coated with silica. Figure 2 shows the TEM images at different magnification of the CNT@Pt@SiO ${ }_{2}$ nanocomposites, offering a $15 \mathrm{~nm}$ thick silica shell. The high homogeneity of the coating allows the silica shell to maintain its thickness even around the aggregates of Pt NPs previously mentioned. The silica coating was carried out in two different deposition steps; considering first the hydrolysis and condensation of 3aminopropyltrimethoxy-silane (APS) and tetraethoxysilane (TEOS), carried out under acidic conditions and consequently under basic conditions. Subsequently, the solution was aged for $10 \mathrm{~h}$ and a compact, uniform, $15 \mathrm{~nm}$ thick silica shell was obtained.

The as-prepared composite nanostructures based on CNTs and Pt NPs and coated with silica were heated up to 550 and $800^{\circ} \mathrm{C}$ in air atmosphere, in two independent experiments. In order to follow the carbonization processes of the composite nanostructures that take place under these conditions, a thermogravimetric analysis (TGA) was performed. Figure 3 includes the TGA as a function of temperature that indicates $\sim 97.7 \mathrm{wt} . \%$ left in the sample tested, in this case after heating up to $830^{\circ} \mathrm{C}$ in air. The TGA analysis offers different steps in the carbonization that can be attributed to the citrate ions surrounding the Pt NPs, the $\mathrm{PAH}$ wrapping the CNTs, and the CNTs themselves, which

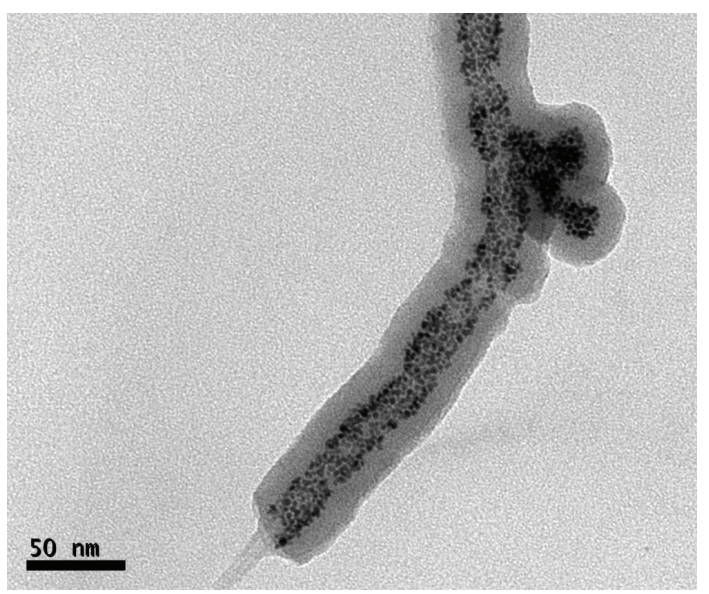

(a)

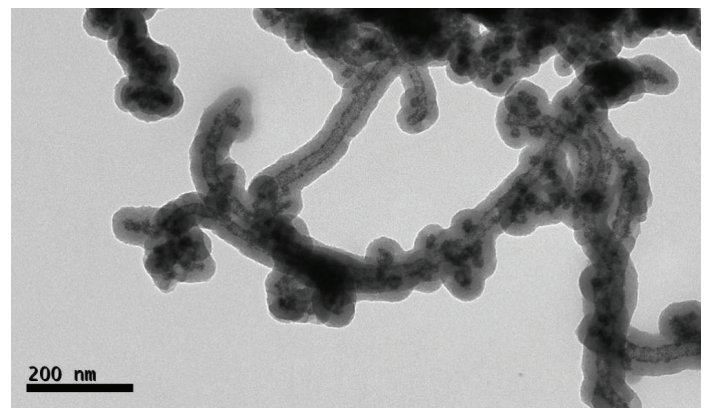

(b)

FIgURE 2: TEM images of CNTs coated with a $15 \mathrm{~nm}$ thick silica shell.

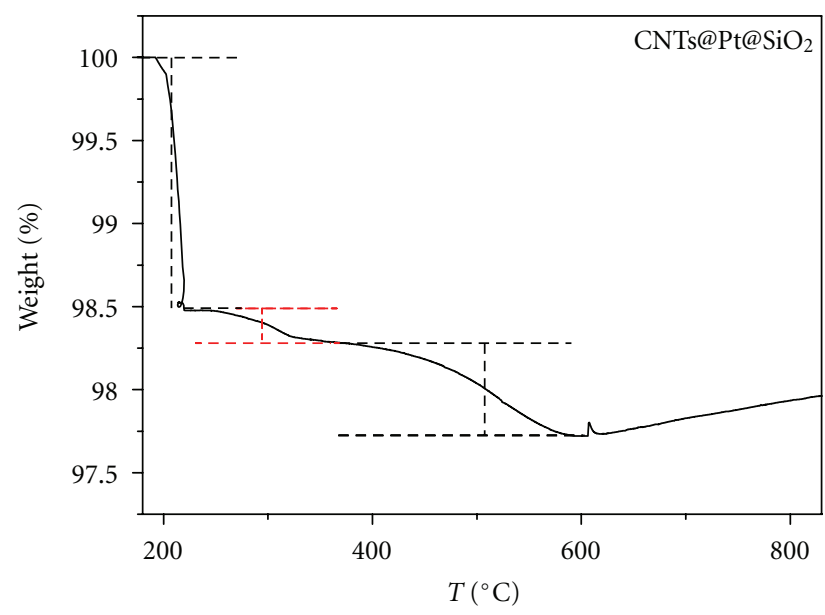

FIGURE 3: TGA analysis while heating the composite nanostructures up to $830^{\circ} \mathrm{C}$.

in air, have been reported to completely decompose after the temperature reached $500^{\circ} \mathrm{C}$ [16], justifying therefore the wt.\% losses. In order to understand this rather low weightreduction, it should be taken into account the extraordinary lightness of CNTs and the branched nature of PAH (that renders possible the wrapping around the CNTs). Both the carbon support and the polyelectrolyte exhibit notably low 


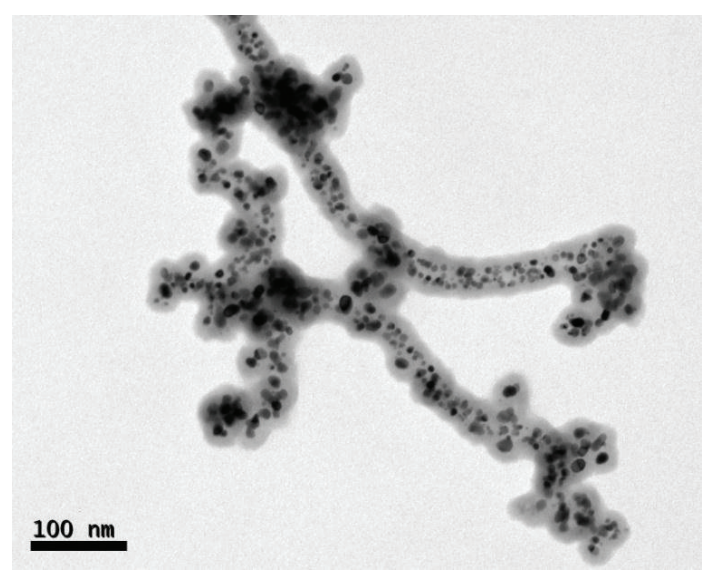

(a)

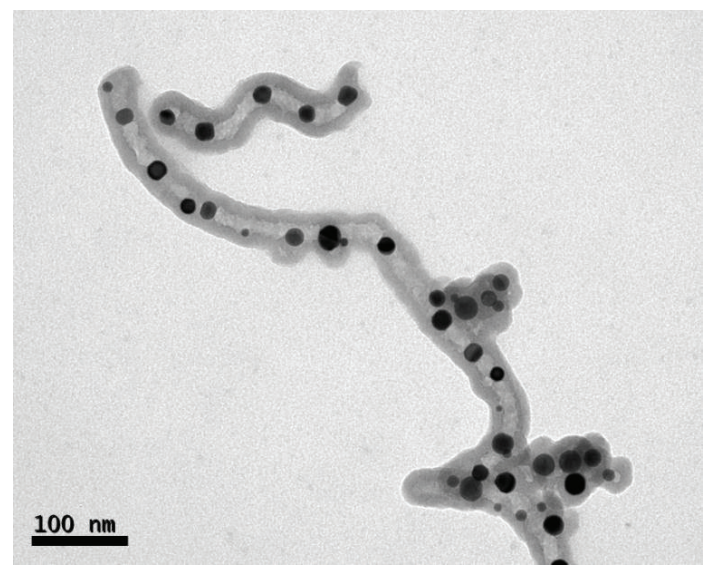

(c)

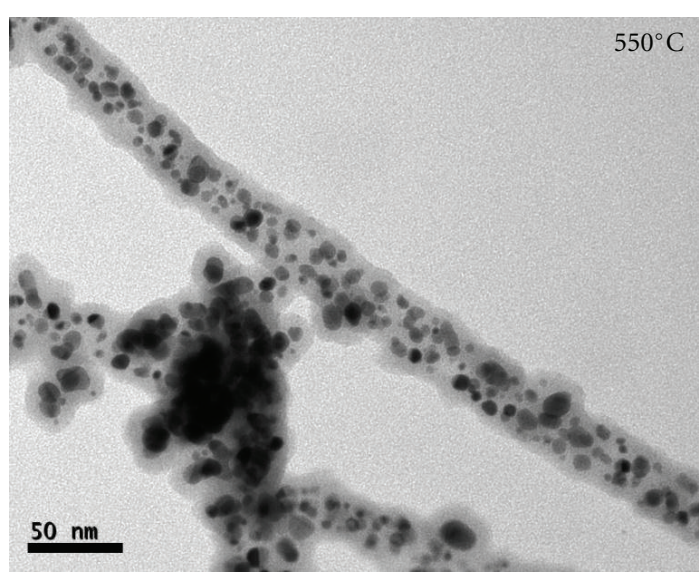

(b)

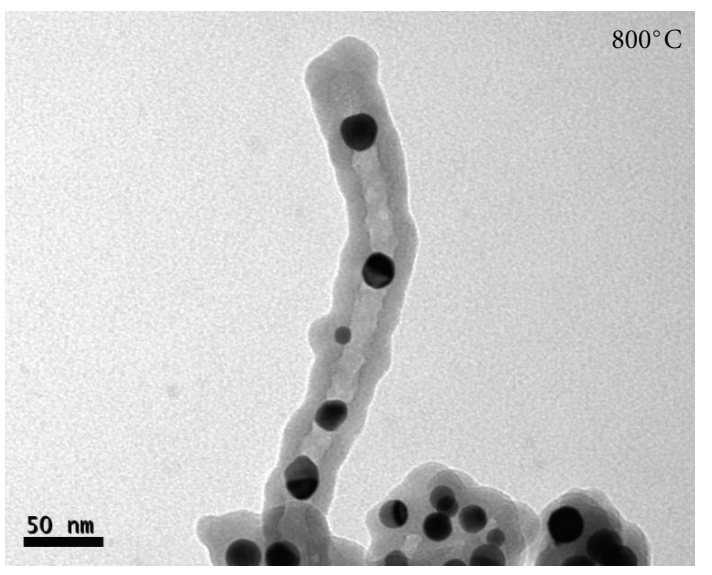

(d)

FIGURE 4: TEM images of the as-prepared composite nanostructures based on CNTs and Pt NPs and coated with silica, heated up to $550^{\circ} \mathrm{C}$ ((a) and (b)) and to $800^{\circ} \mathrm{C}((\mathrm{c})$ and (d)).

densities, which results in the considerably low wt.\% losses mentioned.

After heating up to 550 and $800^{\circ} \mathrm{C}$ in two independent experiments, TEM analysis of the two samples tested was also performed. Figures 4(a) and 4(b) include TEM images of the nanocomposites after heated up to $550^{\circ} \mathrm{C}$, while Figures $4(\mathrm{c})$ and $4(\mathrm{~d})$ show those heated up to $800^{\circ} \mathrm{C}$, reflecting clear changes in the composite nanostructures morphology and in the size of the Pt NPs initially employed.

Clearly, the inner structure of the composites has changed, with a heterogeneous and broader distribution of bigger Pt NPs, not anymore supported onto the CNTs. At $550^{\circ} \mathrm{C}$ the carbonization processes of likely all the organic compounds has already taken place (according to the TGA included in Figure 3) and consequently, no organic frame maintains the NPs in the cylinder-like morphology as when surrounding the CNTs. In this regard, the temperature has started two processes: the carbonization of the organic material and the diffusion [17] of the $\mathrm{Pt}$ atoms that initially formed part of the $2.62 \mathrm{~nm}$ NPs. Considering the increased surface-to-volume ratio of such small Pt NPs, a nonequilibrium process is likely to start as temperature increases, in such a way that the system tries to restore the equilibrium by forming bigger aggregates. Pt atoms initiate therefore a migration to meet again allowing the growth of the bigger NPs. In this sense, the previously formed aggregates of NPs may act as nuclei where to start a growth process. Nevertheless, at $\mathrm{T}<550^{\circ} \mathrm{C}$ the migration process may be slow enough (small migration distances), so that Pt atoms deposit shortly after starting their migration. This situation favors the formation of a broader size distribution $(8.58 \pm 2.45 \mathrm{~nm}$ ( $95 \%$ of NPs) ) of bigger particles, as shown in Figures 4(a) and 4(b). Hence, we can consider the simultaneous dissolution and reaggregation of the NPs, known as Ostwald ripening or coarsening [18]. Since the $2.62 \mathrm{~nm}$ particles are no more stabilized by the citrate ions, already carbonized due to the important increase in temperature and reflected by the TGA analysis (Figure 3), larger nanoparticles are formed at the expense of dissolving smaller ones [19]. These considerations agree with a slow growth rate, which also yields broad size distributions [20].

Figures 4(c) and 4(d) show TEM images of the composites after heated up to $800^{\circ} \mathrm{C}$. The interior of the now formed silica cylinders appears completely clean (compared, 


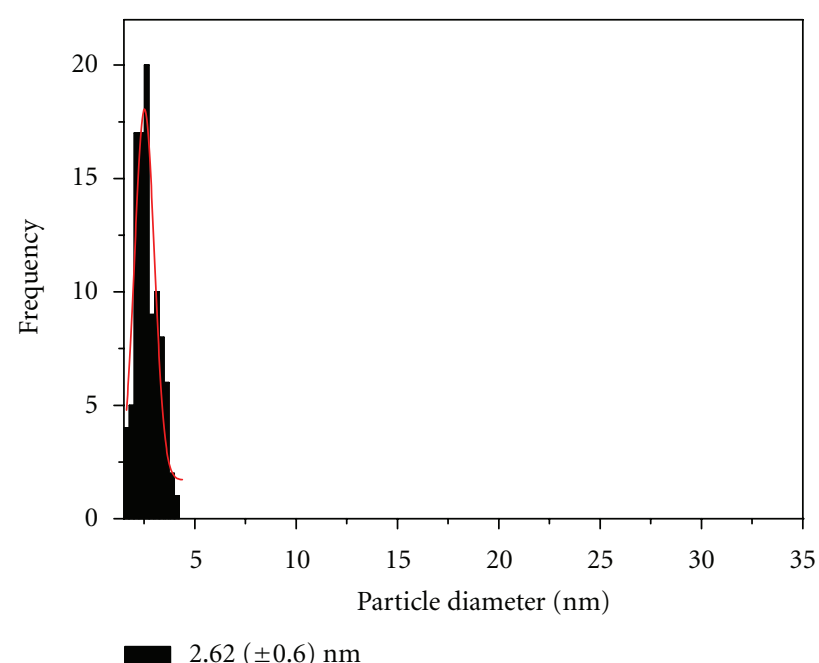

(a)

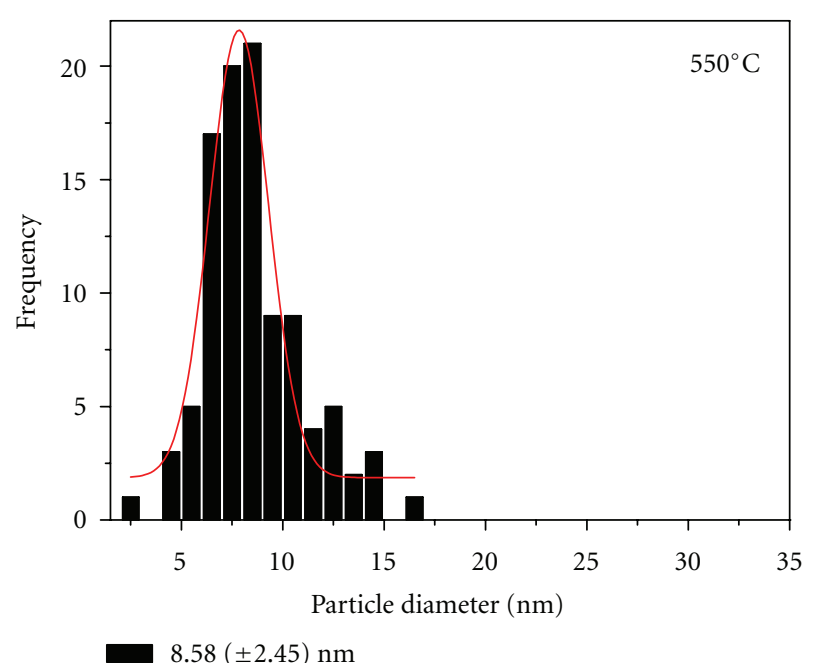

(b)

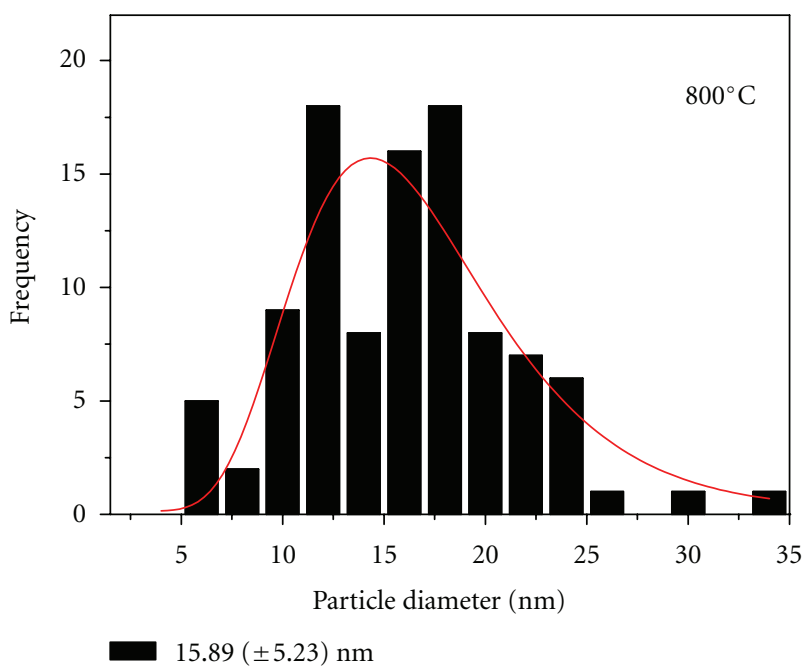

(c)

Figure 5: Average size distributions of the Pt NPs based on TEM images of the samples heated up to 550 and $800^{\circ} \mathrm{C}$.

for example, with the turbid nature of the material appearing in Figures 4(a) and 4(b)), without any organic remains (from the CNTs, citrate ions and PEs) that likely have sublimed. Simultaneously, the silica has compacted leaving an empty inner space in the composite nanostructures. The Pt NPs have now grown until reaching an average size distribution of $15.89 \pm 5.23 \mathrm{~nm}$ ( $95 \%$ of NPs).

According to the migration of atoms proposed with an outcome determined by the diffusion and the deposition flux, this process would then end when atoms have hit NPs of size larger than the critical, that is, stable, in order to condense on them. With increasing sizes, the NPs become more and more stable and impinging atoms condense solely at the bigger ones. In the present case, temperature triggers the migration of the atoms. At low temperatures, migration average distances are small, so that a high density of stable bigger NPs can grow. With increasing temperature, the migration distance also increases and larger and far apart nanoparticles can grow, as shown in the TEM images of Figures 4(c) and 4(d). The TEM images also reveal the increase in the mean particle size due to disappearance of the smaller ones.

The evolution in the particle size distribution reflected by the TEM images is related to the sintering process, described statistically in the light of a simple kinetic model for ripening. Indeed, the heat treatment resulted in a general increase in size and an improvement in the spherical shape, consistent with surface energy minimization. Figure 5 includes the size distribution of the Pt NPs: initially (a), after heating the composites up to $550^{\circ} \mathrm{C}$ (b) and up to $800^{\circ} \mathrm{C}$ (c). The initial Gaussian shape of the average size distribution, that also works for the NPs after heating up to $550^{\circ} \mathrm{C}$ (though broader), varies in the third case (after heating up to $800^{\circ} \mathrm{C}$ ) to a log-normal fit, as a tail of larger NPs emerges on the right side of the mean value, consequently broadening the average size distribution. 
TEM images and resulting average size distributions, indicating that smaller NPs have eventually disappeared and larger ones have been obtained, are consistent with a sintering process of Pt NPs governed by an Ostwald ripening mechanism. The corresponding mass-transport can be justified if mediated by $\mathrm{Pt}$ atoms, or more likely by Ptoxygen species (the sintering rate of Pt NPs in oxidative environments was reported to be accelerated due to the formation of volatile Pt-oxygen species)[21]. Besides the oxygen-rich environment, we have to take into account the increased temperatures reached and the fact that Pt NPs are neither free nor supported on planar substrates but confined in the cylindrical empty spaces given once the carbonization of CNTs and the compaction of silica are accomplished. Consequently, a local effect favoring the exchange rate of diffusing species between NPs is very much favored.

\section{Conclusions}

Transmission electron microscopy has been employed in order to monitor the evolution of Pt NPs deposited onto the surface of carbon nanotubes while heating independently up to 550 and $800^{\circ} \mathrm{C}$. These experiments reveal unequivocally a favored Ostwald-ripening-operated sintering process taking place in the CNT/silica-templated confined space formed as temperature is raised.

\section{Acknowledgments}

C. Mateo-Mateo and V. Salgueiriño acknowledge the financial support from the F.P.U. and Ramón y Cajal Program (Ministerio de Ciencia e Innovación, Spain). M. PérezLorenzo acknowledges the financial support from the Isidro Parga Pondal Program (Xunta de Galicia, Spain). This work has been supported by the Xunta de Galicia Regional Government (INCITE09209101PR, INCITE-08PXIB209007PR, 2008/077, and 2010/78).

\section{References}

[1] R. T. K. Baker, C. H. Bartholomew, and D. B. Dadyburjor, Sintering and Redispersion: Mechanisms and Kinetics. Stability of Supported Catalysts: Sintering and Redispersion, Edited by J. A. Horsley, Catalytica, Mountain View, Calif, USA, 1991.

[2] S. B. Simonsen, I. Chorkendorff, S. Dahl et al., "Effect of particle morphology on the ripening of supported $\mathrm{Pt}$ nanoparticles," Journal of Physical Chemistry C, vol. 116, no. 9, pp. 5646-5653, 2012.

[3] S. R. Challa, A. T. Delariva, T. W. Hansen et al., "Relating rates of catalyst sintering to the disappearance of individual nanoparticles during Ostwald ripening," Journal of the American Chemical Society, vol. 133, no. 51, pp. 20672-20675, 2011.

[4] P. Wynblatt and N. A. Gjostein, "Supported metal crystallites," Progress in Solid State Chemistry, vol. 9, pp. 21-58, 1975.

[5] F. Yang, M. S. Chen, and D. W. Goodman, "Sintering of Au particles supported on $\mathrm{TiO}_{2}(110)$ during CO oxidation," Journal of Physical Chemistry C, vol. 113, no. 1, pp. 254-260, 2009.

[6] S. B. Simonsen, I. Chorkendorff, S. Dahl, M. Skoglundh, J. Sehested, and S. Helveg, "Direct observations of oxygeninduced platinum nanoparticle ripening studied by in situ
TEM," Journal of the American Chemical Society, vol. 132, no. 23, pp. 7968-7975, 2010.

[7] M. Sanlés, M. Pérez-Lorenzo, B. Rodríguez-González, V. Salgueiriño, and M. A. Correa-Duarte, "Highly active nanoreactors: nanomaterial encapsulation based on confined catalysis," Angewandte Chemie International Edition, vol. 51, pp. 3877-3882, 2012.

[8] M. de Dios, V. Salgueirino, M. Pérez-Lorenzo, and M. A. Correa-Duarte, "Synthesis of carbon nanotube-inorganic hybrid nanocomposites: an instructional experiment in nanomaterials chemistry," "Journal of Chemical Education, vol. 89, pp. 280-283, 2012.

[9] M. Grzelczak, M. A. Correa-Duarte, and L. M. Liz-Marzán, "Carbon nanotubes encapsulated in wormlike hollow silica shells," Small, vol. 2, no. 10, pp. 1174-1177, 2006.

[10] A. A. Dameron, S. Pylypenko, J. B. Bult et al., "Aligned carbon nanotube array functionalization for enhanced atomic layer deposition of platinum electro-catalysts," Applied Surface Science, vol. 258, no. 13, pp. 5212-5221, 2012.

[11] M. J. O’Connell, P. Boul, L. M. Ericson et al., "Reversible water-solubilization of single-walled carbon nanotubes by polymer wrapping," Chemical Physics Letters, vol. 342, no. 3-4, pp. 265-271, 2001.

[12] M. A. Correa-Duarte and L. M. Liz-Marzán, "Carbon nanotubes as templates for one-dimensional nanoparticle assemblies," Journal of Materials Chemistry, vol. 16, no. 1, pp. 22-25, 2006.

[13] S. Kawasaki, G. Catalan, H. J. Fan et al., "Conformal oxide coating of carbon nanotubes," Applied Physics Letters, vol. 92, no. 5, Article ID 053109, 2008.

[14] M. A. Correa-Duarte, N. Sobal, L. M. Liz-Marzán, and M. Giersig, "Linear assemblies of silica-coated gold nanoparticles using carbon nanotubes as templates," Advanced Materials, vol. 16, no. 23-24, pp. 2179-2184, 2004.

[15] F. Rivadulla, C. Mateo-Mateo, and M. A. Correa-Duarte, "Layer-by-layer polymer coating of carbon nanotubes: tuning of electrical conductivity in random networks," Journal of the American Chemical Society, vol. 132, no. 11, pp. 3751-3755, 2010.

[16] R. B. Mathur, S. Seth, C. Lal et al., "Co-synthesis, purification and characterization of single- and multi-walled carbon nanotubes using the electric arc method," Carbon, vol. 45, no. 1, pp. 132-140, 2007.

[17] H. Rôder, E. Hahn, H. Brune, J. P. Bucher, and K. Kern, "Building one- and two-dimensional nanostructures by diffusioncontrolled aggregation at surfaces," Nature, vol. 366, no. 6451, pp. 141-143, 1993.

[18] M. Bowker, "Surface science: the going rate for catalysts," Nature Materials, vol. 1, no. 4, pp. 205-206, 2002.

[19] F. Huang, H. Zhang, and J. F. Banfieldt, "Two-stage crystalgrowth kinetics observed during hydrothermal coarsening of nanocrystalline ZnS," Nano Letters, vol. 3, no. 3, pp. 373-378, 2003.

[20] Y. Yin and A. P. Alivisatos, "Colloidal nanocrystal synthesis and the organic-inorganic interface," Nature, vol. 437, no. 7059, pp. 664-670, 2005.

[21] P. Loof, B. Stenbom, H. Norden, and B. Kasemo, "Rapid sintering in $\mathrm{NO}$ of nanometer-sized $\mathrm{Pt}$ particles on $\gamma-\mathrm{Al}_{2} \mathrm{O}_{3}$ observed by $\mathrm{CO}$ temperature-programmed desorption and transmission electron microscopy," Journal of Catalysis, vol. 144, no. 1, pp. 60-76, 1993. 

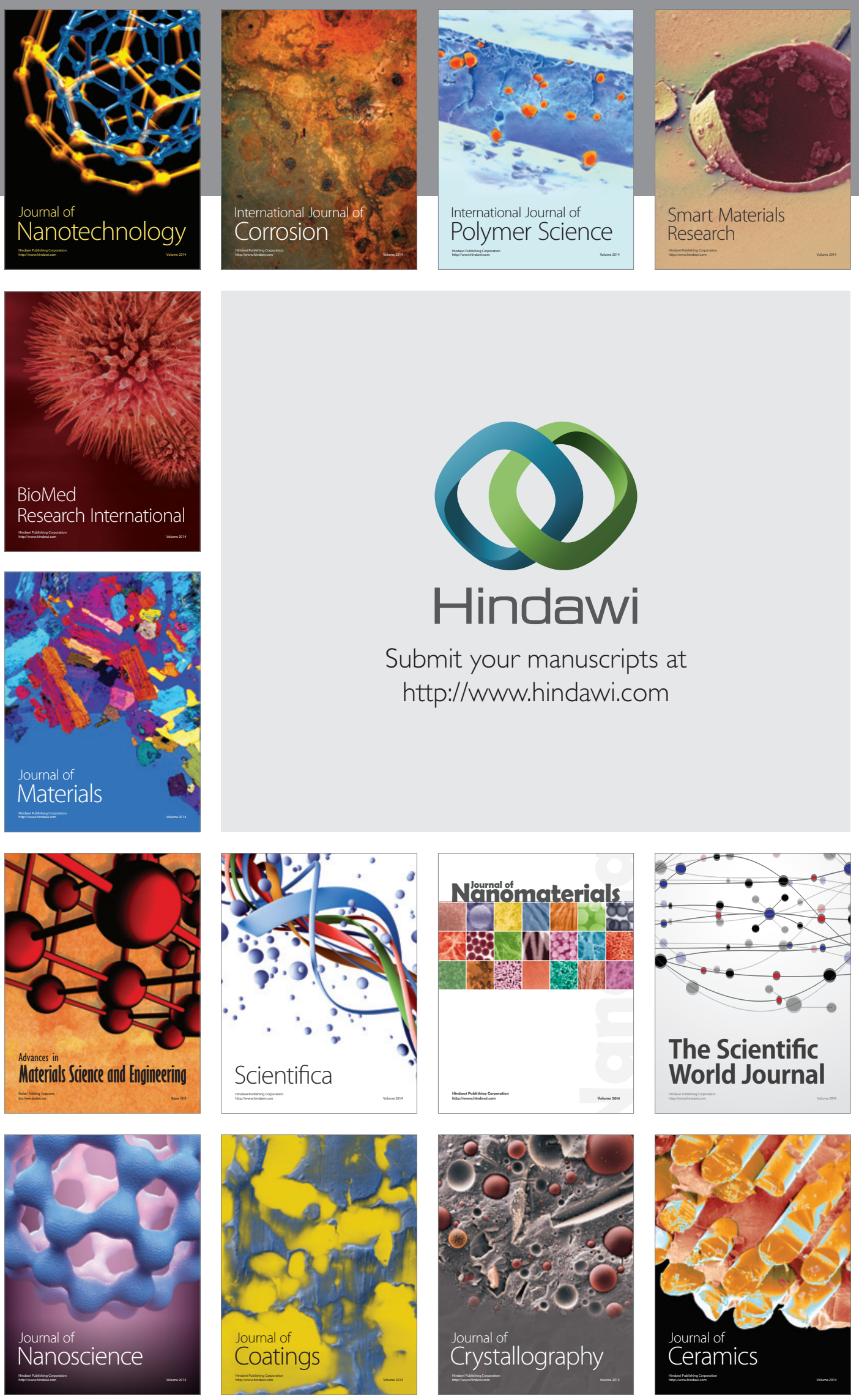

The Scientific World Journal

Submit your manuscripts at

http://www.hindawi.com

\section{World Journal}

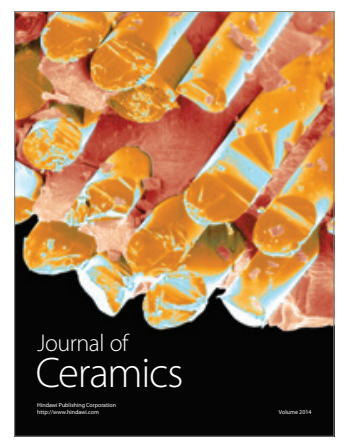

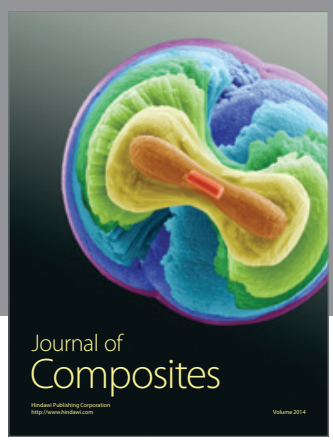
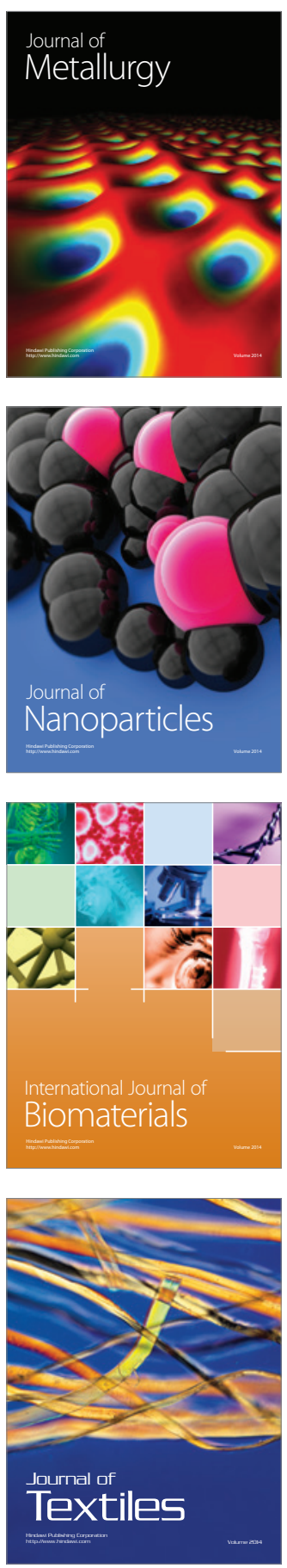